\title{
Norcantharidin inhibits IL-6-induced epithelial-mesenchymal transition via the JAK2/STAT3/TWIST signaling pathway in hepatocellular carcinoma cells
}

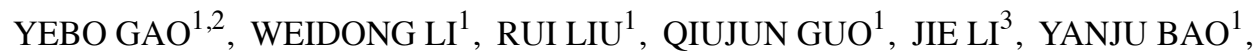 \\ HONGGANG ZHENG ${ }^{1}$, SHULONG JIANG ${ }^{1,3}$ and BAOJIN HUA ${ }^{1}$ \\ ${ }^{1}$ Department of Oncology, Guang'anmen Hospital, China Academy of Chinese Medical Sciences, Beijing 100053; \\ ${ }^{2}$ Department of Oncology, Wangjing Hospital, China Academy of Chinese Medical Sciences, Beijing 100102; \\ ${ }^{3}$ Department of Oncology, Jining First People's Hospital, Jining, Shandong 272111, P.R. China
}

Received November 20, 2016; Accepted June 19, 2017

DOI: 10.3892/or.2017.5775

\begin{abstract}
Epithelial-mesenchymal transition (EMT), plays a vital role in hepatocellular carcinoma (HCC) development and metastasis. Norcantharidin (NCTD; 7-oxabicyclo (2.2.1) heptane-2,3-dicarboxylic anhydride) plays anticancer roles in the regulation of tumor cell proliferation, apoptosis and migration. However, the molecular mechanism of HCC EMT and the effects of NCTD in the HCC EMT process have been either poorly elucidated or not studied. In this study, HCC EMT was induced by the treatment of IL- 6 and various concentrations of $\operatorname{NCTD}(0,30,60$ and $120 \mu \mathrm{M})$ were treated with HCC cell lines, HCCLM3 and SMMC-7721. We investigated the effect of NCTD on the invasion of HCC cells by using Transwell assay. Immunofluorescence staining, western blot analysis and quantitative RT-PCR were performed to evaluate the protein and mRNA expression levels of HCC cells. Here, using cell line models, our data demonstrated that interleukin 6 (IL-6) induced EMT through the JAK/STAT3/TWIST pathway in HCC. Moreover, our studies revealed that NCTD markedly inhibited IL-6-induced EMT and cell invasiveness. Signaling studies revealed that NCTD sufficiently suppressed JAK/ STAT3/TWIST signaling to reverse the IL-6-promoting effects. Collectively, these data provide evidence for the use of NCTD as a potential anticancer drug in HCC metastatic patients.
\end{abstract}

Correspondence to: $\mathrm{Dr}$ Shulong Jiang or Dr Baojin Hua, Department of Oncology, Guang'anmen Hospital, China Academy of Chinese Medical Sciences, Beijing 100053, P.R. China

E-mail: jnsljiang@163.com

E-mail: huabaojin@sohu.com

Key words: norcantharidin, epithelial-mesenchymal transition, JAK2/STAT3/TWIST, hepatocellular carcinoma

\section{Introduction}

Hepatocellular carcinoma (HCC) is the fifth most commonly diagnosed cancer and second most frequent cause of cancer-related deaths for males worldwide. In women, it is the seventh most commonly diagnosed cancer and the sixth leading cause of cancer-related deaths (1). HCC is associated with a poor prognosis due to late diagnosis and a lack of effective treatment options. Although great advances in surgical techniques and medical care have been achieved over the last several decades, the 5-year survival rate worldwide of HCC is still less than $5 \%$, mainly due to the high rate of recurrence and metastasis (2). In fact, increasing evidence suggests that HCC metastasis is a multistep process. During progression to metastasis, cancer cells are thought to acquire a mesenchymal phenotype, which allows them to leave the site of the primary tumor, invade surrounding tissues, and migrate to distant organs. After seeding, these cells switch back to an epithelial phenotype and proliferate to form metastases $(3,4)$. The process by which cells switch from epithelial-mesenchymal (EMT) phenotypes is known as EMT transition (5). EMT is a critical prognostic factor in $\mathrm{HCC}$ and is involved in early recurrence or metastases after surgery (6). Blocking EMT is currently considered as a promising strategy to inhibit cancer metastasis and improve patient survival.

Growing evidence suggests that inflammation promotes EMT $(7,8)$. Interleukin 6 (IL-6) a multifunctional cytokine in the tumor microenvironment, has been regarded as the main factor involved in EMT, contributing to tumor invasion and metastasis (9-11). Previous studies revealed that IL-6 leads to the development of $\mathrm{HCC}(12,13)$, as an independent predictor of HCC tumor recurrence, poor survival, and tumor metastasis (14). Furthermore, IL-6 appears to contribute as a potent factor to the initiation of EMT via activation of Janus kinase (JAK) family members (JAK1, JAK2, and TYK2), leading to the activation of transcription factors of the signal transducer and activator of transcription 3 (STAT3) signaling (15-17). Emerging evidence suggests that STAT3 regulated the expression of Twist by directly binding to the transcriptional starting site in the Twist promoter (18). Moreover, blocking STAT3 
markedly suppressed the expression of Twist, confirming the regulation of Twist by STAT3 (17). However, little is known about the mechanisms of IL-6-induced EMT in HCC.

Increasing evidence has indicated that traditional Chinese medicines contain anticancer ingredients. Norcantharidin (NCTD; 7-oxabicyclo (2.2.1) heptane-2,3-dicarboxylic anhydride) is a demethylated and low-cytotoxic analog of cantharidin, an active ingredient of the Chinese blister beetle Mylabris, which has been used in China to treat tumors, inflammation and many other conditions for a long time $(19,20)$. Previous studies have reported that NCTD could modulate the expression of Bcl-2 and Mcl-1 to effectively inhibit proliferation and induce apoptosis in a variety of human tumor cells, as well as producing fewer side effects and leukocytosis (21-27). Several studies have suggested that NCTD inhibits cell migration and invasion through the c-Jun N-terminal kinase (JNK) and mitogen-activated protein (MAP) kinase signaling pathways in human lung cancer cells and CRC cells (28-30). However, the effects of NCTD on HCC metastasis have not been elucidated thus far.

In the present study, we investigated the anti-metastatic effects of NCTD using IL-6-treated HCC cells. We found that NCTD inhibited IL-6-induced EMT and invasiveness through the suppression of the JAK2 and STAT3 pathways to regulate TWIST expression in HCC cell lines.

\section{Materials and methods}

Compound preparations and reagents. NCTD (molecular formular: $\mathrm{C}_{8} \mathrm{H}_{8} \mathrm{O}_{4}$, molecular weight: 168.1467, HPLC $\geq 98 \%$ ) was purchased from the National Standard Network (Beijing, China). NCTD was dissolved in dimethyl sulphoxide (DMSO) to a concentration of $40 \mathrm{mg} / \mathrm{ml}$ as stock solution, stored at room temperature and protected from the light. Serial concentrations of NCTD were diluted in culture medium before use (DMSO <1\%, 0-120 $\mu \mathrm{M}$ ). Cucurbitacin I (JSI-124, \#C4493-1MG; Sigma-Aldrich, St. Louis, MO, USA) a novel inhibitor of the JAK2/STAT3 signaling pathway (molecular weight: 514.65 , HPLC $\geq 95 \%$ ) (31), was dissolved in DMSO to a concentration of $5 \mathrm{mg} / \mathrm{ml}$ as stock solution, stored at $-20^{\circ} \mathrm{C}$ and protected from the light. Then, cucurbitacin I was diluted to a concentration of $0.5 \mu \mathrm{M}$ in culture medium before use (DMSO <1\%). Human recombinant IL-6 (\#I1395-50UG; Sigma-Aldrich) was dissolved in phosphate-buffered saline (PBS) to a concentration of $100 \mu \mathrm{g} / \mathrm{ml}$ as stock solution and stored at $-20^{\circ} \mathrm{C}$. The final concentration of IL- 6 was diluted to $100 \mathrm{ng} / \mathrm{ml}$ in culture medium before use.

Cell culture. HCC cell lines, HCCLM3 and SMMC-7721, were purchased from the China Infrastructure of Cell Line Resources (Beijing, China). Cells were cultured in Dulbecco's modified Eagle's medium (Gibco, Grand Island, NY, USA) supplemented with $10 \%$ heat-inactivated fetal bovine serum (Hyclone, Logan, Utah, USA) and 1\% penicillin and streptomycin (Hyclone) in a $5 \% \mathrm{CO}_{2}$ humidified incubator at $37^{\circ} \mathrm{C}$.

Cell invasion assays. Transwell Matrigel invasion chambers (\#3422; Corning Costar Corporation, USA) with $8-\mu \mathrm{m}$ membrane pores coated with $100 \mu 1$ of 1:6 diluted Matrigel in serum-free DMEM (\#356234; BD Biosciences, CA, USA) were used for the cell invasion assay. Cells were pretreated with $100 \mathrm{ng} / \mathrm{ml}$ of IL-6 for $48 \mathrm{~h}$. Cells $\left(1 \times 10^{4}\right)$ were plated in $100 \mu \mathrm{l}$ of serum-free DMEM containing NCTD $(0,30,60$ and $120 \mu \mathrm{M})$ and JSI-124 $(0.5 \mu \mathrm{M})$ as the positive control group into the upper chamber. The lower chamber was filled with $600 \mu \mathrm{l}$ of DMEM and 10\% FBS medium. After incubation at $37^{\circ} \mathrm{C}$ for $24 \mathrm{~h}$ in a $5 \% \mathrm{CO}_{2}$ atmosphere, the non-invaded cells in the inserts were removed with cotton swabs. The invaded cells on the underside were treated with a fixative/staining solution ( $0.1 \%$ crystal violet, $4 \%$ paraformaldehyde) for visualization. The number of invading cells on the filters was counted in 5 random fields per filter at an x100 magnification in triplicate wells for each group.

Immunofluorescence staining. Cells were treated with $100 \mathrm{ng} / \mathrm{ml}$ of IL-6 and grown in 96-well plates. The cells were fixed for 15 min with $4 \%$ paraformaldehyde, and permeabilized for 10 min in PBS containing 0.1\% Triton X-100 at room temperature. The cells were rinsed in PBS (pH 7.4) three times for 5 min. After blocking in Immunol staining blocking buffer (Beyotime Biotechnology, Shanghai, China) for $1 \mathrm{~h}$ at room temperature, the cells were probed with a primary antibody at $4^{\circ} \mathrm{C}$ overnight. After rinsing in PBS, the cells were incubated with secondary antibodies [anti-rabbit IgG-Alexa Fluor 555-conjugated (red) or anti-rabbit IgG-Alexa Fluor 488-conjugated (green) ] for $1 \mathrm{~h}$ at room temperature and the nuclei were stained with DAPI (Beyotime Biotechnology) for $10 \mathrm{~min}$. All of the images were semi-quantitatively analyzed, and the 96-well plates were mounted and viewed using ImageXpress Micro (high content analysis; Molecular Devices, CA, USA). For analysis of E-cadherin, N-cadherin and vimentin expression, fluorescence intensity was quantified by assessing the intensity in the cells using Image-Pro Plus software, version 6.0 (Media Cybernetics, Bethesda, MD, USA).

Western blot analysis. HCCLM3 cells were pretreated with $100 \mathrm{ng} / \mathrm{ml}$ of IL-6 for $48 \mathrm{~h}$ and then treated with NCTD (0, 30,60 , and $120 \mu \mathrm{M}$ ) for $24 \mathrm{~h}$. The positive control group was subjected to JSI-124 treatment (JAK2/STAT3 inhibitor, $0.5 \mu \mathrm{M}$ ) for $24 \mathrm{~h}$. The cells were lysed in a RIPA buffer with a protease inhibitor cocktail and a phosphatase inhibitor cocktail (both from Beyotime Biotechnology), and then clarified by centrifugation. Total cell lysates were resuspended in SDS sample buffer and resolved by SDS-PAGE. Proteins were transferred to polyvinylidene fluoride (PVDF) membranes (Millipore Corp., Billerica, MA, USA), and then blocked with $5 \%$ non-fat milk for $1 \mathrm{~h}$ at room temperature. The membranes were then incubated with primary antibodies [E-cadherin (\#3195), N-cadherin (\#13116), vimentin (\#5741), JAK2 (\#3230), pJAK2 (Tyr1007/1008,\#3776), STAT3 (\#12640) and pSTAT3 (Tyr705,\#9145), (all from Cell Signaling Technology, Inc., Danvers, MA, USA), and Twist (H-81, \#sc-15393; Santa Cruz Biotechnology, Dallas, Texas, USA)], overnight at $4^{\circ} \mathrm{C}$ before incubation with the corresponding HRP-conjugated secondary antibodies $(1: 10,000)$ diluted in TBST. Then, the membranes were washed extensively with TBST and visualized using an enhanced chemiluminescence detection system, followed by quantification using the Image Quant LAS 4000 (GE Healthcare, Bucks, UK). 


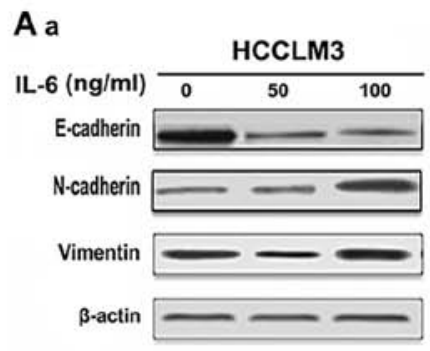

b

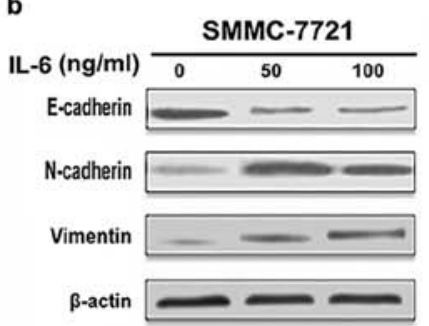

C a

b
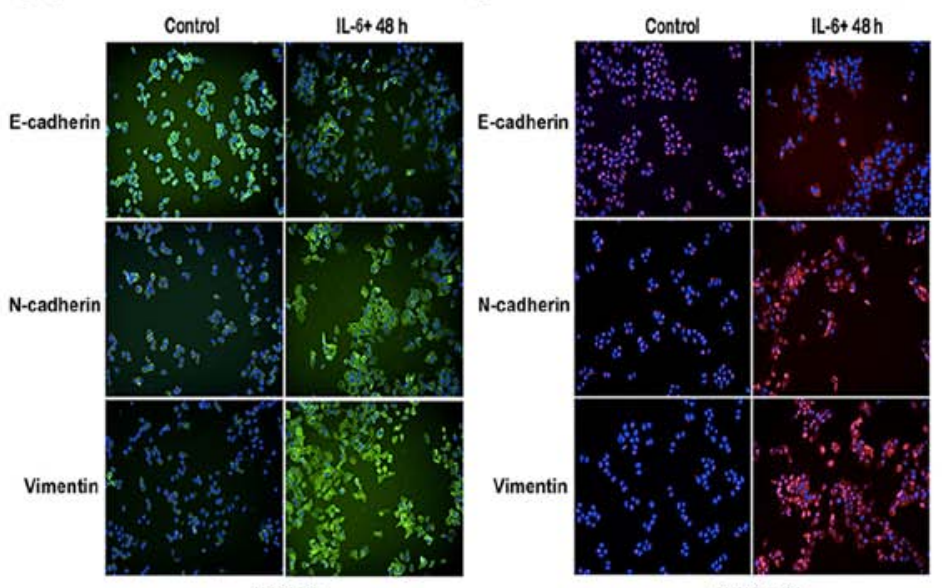

SMMC-7721
B a

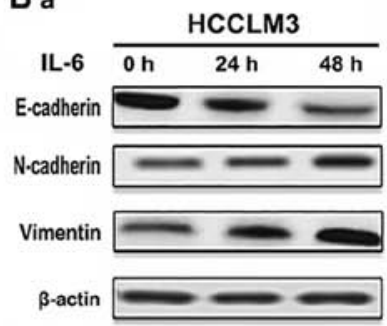

b

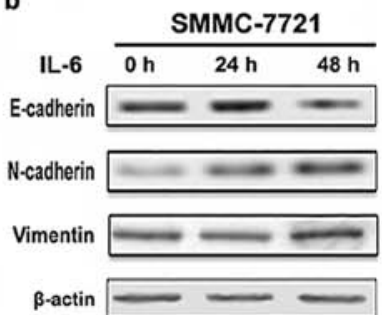

D a

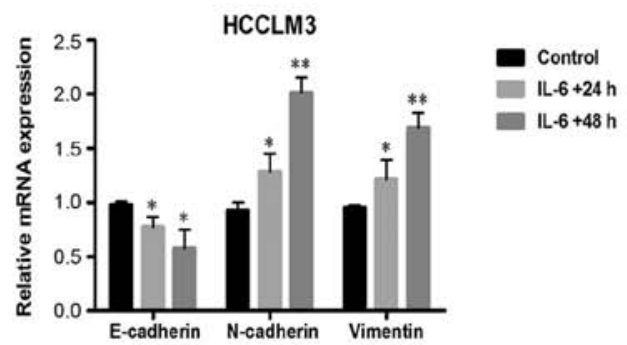

b

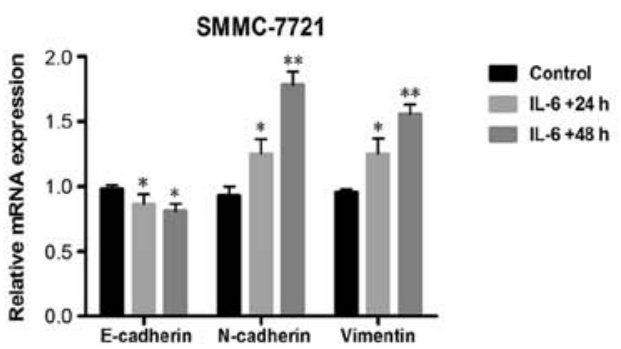

Figure 1. IL-6 treatment induces EMT in HCC cell lines. (A-a and b) Cells were treated with IL-6 (0, 50 and $100 \mathrm{ng} / \mathrm{ml})$ for 48 h.The protein levels of EMT markers were assessed by western blot analysis in HCCLM3 and SMMC-7721 cell lines. (B-a and b) Cells were treated with IL-6 (100 ng/ml) for 0, 24 and $48 \mathrm{~h}$. The protein levels of EMT markers were assessed by western blot analysis in HCCLM3 and SMMC-7721 cell lines. (C-a) HCCLM3 cells were treated without or with IL-6 $(100 \mathrm{ng} / \mathrm{ml})$ for $48 \mathrm{~h}$. Cells were stained with primary antibodies against E-cadherin, $\mathrm{N}$-cadherin and vimentin, followed by Alexa Fluor 488-conjugated (green) secondary antibodies. Nuclei were stained with DAPI (blue) (original magnification, x400). (C-b) SMMC-7721 cells were treated without or with IL-6 (100 ng/ml) for $48 \mathrm{~h}$. Cells were stained with primary antibodies against E-cadherin, N-cadherin and vimentin, followed by Alexa Fluor 555-conjugated (red) secondary antibodies. Nuclei were stained with DAPI (blue) (original magnification, x400). (D-a and b) Cells were exposed to $100 \mathrm{ng} / \mathrm{ml}$ of IL-6 for 24 and $48 \mathrm{~h}$. The mRNA expression levels of E-cadherin, N-cadherin and vimentin were determined by quantitative RT-PCR (mean values $\pm \mathrm{SD}, \mathrm{n}=3$, are provided. $\left.{ }^{*} \mathrm{P}<0.05,{ }^{* * *} \mathrm{P}<0.01\right)$. IL-6, interleukin 6; EMT, epithelial-mesenchymal transition; HCC, hepatocellular carcinoma.

Table I. The sequences of primers.

\begin{tabular}{ll}
\hline Target gene & \multicolumn{1}{c}{ Primers sequences } \\
\hline E-cadherin & F: 5'-CCCATCAGCTGCCCAGAAAATGAA-3' \\
& R: 5'-CTGTCACCTTCAGCCATCCTGTTT-3' \\
N-cadherin & F: 5'-AAGAACGCCAGGCCAAACAAC-3' \\
& R: 5'-CTGGCTCAAGTCATAGTCCTGGTCT-3' \\
Vimentin & F: 5'-GACAATGCGTCTCTGGCACGTCTT-3' \\
& R: 5'-TCCTCCGCCTCCTGCAGGTTCTT-3' \\
JAK2 & F:5'-TGGACAGAGAGAGAATTTCTGAACT-3' \\
& R: 5'-TTCATTGCTTTCCTTTTTCACA-3' \\
STAT3 & F: 5'-TTGCCAGTTGTGGTGATC-3' \\
& R: 5'-AGAACCCAGAAGGAGAAGC-3' \\
TWIST & F: 5'-GTCCGCAGTCTTACGAGGAG-3' \\
& R: 5'-GCTTGAGGGTCTGAATCTTGCT-3' \\
GAPDH & F: 5'-TGCACCACCAACTGCTTAGC-3' \\
& R: 5'-GGCATGGACTGTGGTCATGAG-3' \\
\hline
\end{tabular}

F, forward; R, reverse; JAK, Janus tyrosine kinase; STAT3, signal transducer and activator of transcription 3 .
Quantitative RT-PCR. Total RNA was extracted from individual groups of cells using TRIzol reagent and reversely transcribed to cDNA using the First Strand cDNA Synthesis kit (Invitrogen). cDNA was synthesized using $1 \mu \mathrm{g}$ of total RNA in a $20-\mu$ l final volume by reverse transcription utilizing SuperScript II Reverse Transcriptase with oligo-dT(18)-primers (both from Invitrogen). The relative levels of target gene mRNA transcripts were determined by quantitative RT-PCR using the SYBR-Green Master Mix kit. RNA was amplified using ABI Prism 7000 Sequence Detection System (Applied Biosystems). The sequences of the primers are shown in Table I. The PCR amplification was performed in triplicate at $95^{\circ} \mathrm{C}$ for $10 \mathrm{~min}$ and was subjected to 40 cycles of $95^{\circ} \mathrm{C}$ for $15 \mathrm{sec}$ and $60^{\circ} \mathrm{C}$ for $30 \mathrm{sec}$. The relative levels of each gene to GAPDH mRNA transcripts were calculated.

Statistical analysis. Data were expressed as the mean \pm standard deviation (SD) and statistical differences were determined by two-way repeated ANOVA or by one-way ANOVA, followed by a Newman-Keuls test using SPSS 13.0 statistical software. A p-value of $<0.05$ was considered significant. 
A a

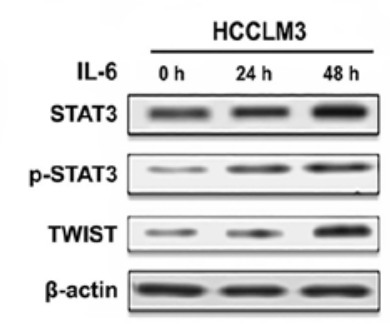

B a

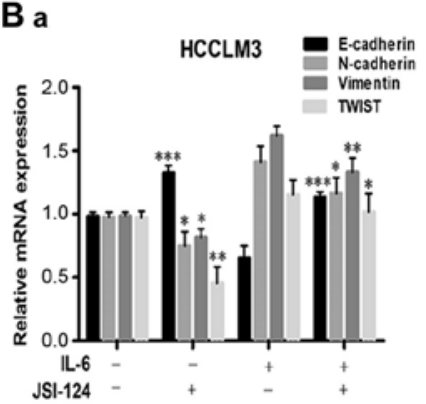

b

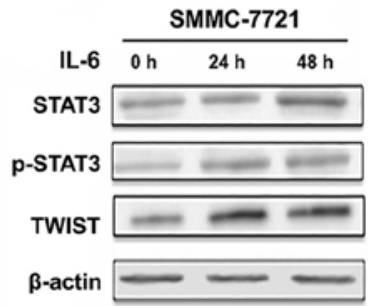

b

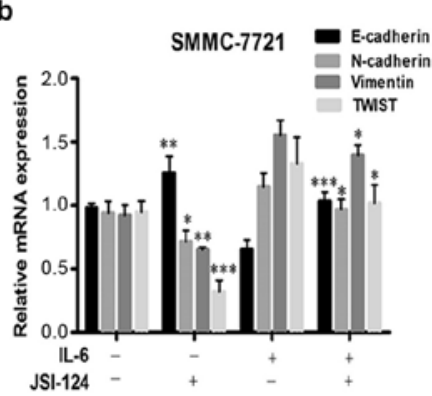

c

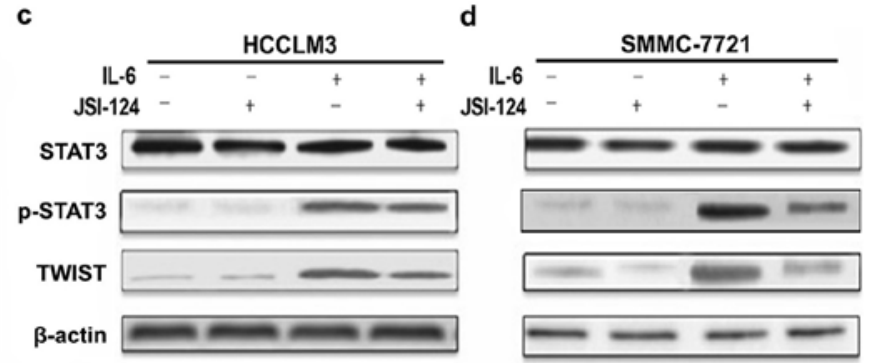

C

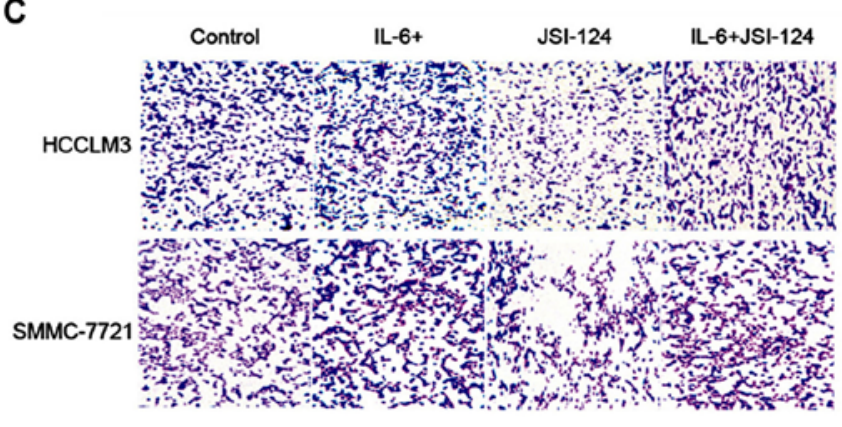

D a

b
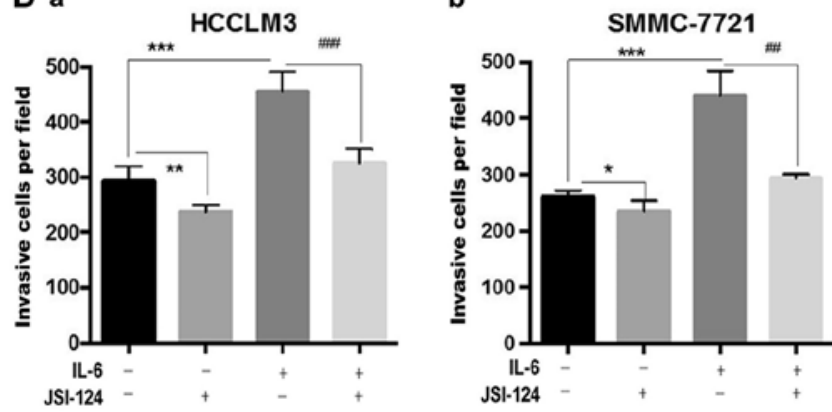

Figure 2. JAK2/STAT3/TWIST signaling is required for IL-6-induced EMT in HCC cell lines.(A-a and b) Western blot analysis of p-STAT3-Y705, STAT3 and TWIST in HCCLM3 and SMMC-7721 cells after IL-6 treatment for the indicated periods (0, 24 and 48 h). (A-c and d) JSI-124 inhibited IL-6-induced STAT3 phosphorylation and TWIST expression in HCCLM3 and SMMC-7721 cell lines. (B-a and b) Cells were pretreated with $100 \mathrm{ng} / \mathrm{ml}$ of IL-6 for $48 \mathrm{~h}$ and then exposed to $0.5 \mu \mathrm{M}$ of JAK2/STAT3 inhibitor (JSI-124) for $24 \mathrm{~h}$. The mRNA levels of E-cadherin, $\mathrm{N}$-cadherin, vimentin and Twist were determined by RT-PCR in HCCLM3 and SMMC-7721 cell lines. JSI-124 significantly reversed IL-6-mediated downregulation of E-cadherin, upregulation of N-cadherin and vimentin, and decreased mRNA levels of Twist. (C and D-a and b) JSI-124 inhibited IL-6-induced cell invasion in HCCLM3 and SMMC-7721 cells. Cells were treated with $100 \mathrm{ng} / \mathrm{ml}$ of IL-6 and/or $0.5 \mu \mathrm{M}$ of JSI- 124 for $24 \mathrm{~h}$, and allowed to pass through $8-\mu \mathrm{m}$ membrane pores coated with $100 \mu \mathrm{l}$ of Matrigel in Transwells. Invasive cells through the pores were stained with $1 \%$ crystal violet (magnification, $x 200$ ). The mean values $\pm S D, n=3$ are provided. "P<0.05, ${ }^{* *} \mathrm{P}<0.01,{ }^{* * *} \mathrm{P}<0.001$ vs. the control group, ${ }^{\#} \mathrm{P}<0.05,{ }^{\# \#} \mathrm{P}<0.01,{ }^{\# \# \#} \mathrm{P}<0.001$ vs. the IL-6+ and JSI-124-). JAK, Janus tyrosine kinase; STAT3, signal transducer and activator of transcription 3; IL-6, interleukin 6; EMT, epithelial-mesenchymal transition; HCC, hepatocellular carcinoma.

\section{Results}

IL-6 induces EMT in HCC cells. To investigate whether recombinant IL-6 could induce EMT in HCC cells, we treated the human HCC cell lines, HCCLM3 and SMMC-7721, which exhibit an epithelial phenotype, with various concentrations $(0,50$, and $100 \mathrm{ng} / \mathrm{ml})$ of recombinant IL-6 for $48 \mathrm{~h}$ or with $100 \mathrm{ng} / \mathrm{ml}$ of IL- 6 for the indicated durations (0, 24, and $48 \mathrm{~h})$. We assessed the expression of epithelial marker, E-cadherin, and the mesenchymal markers, $\mathrm{N}$-cadherin and vimentin. The results demonstrated that E-cadherin expression was significantly suppressed, although $\mathrm{N}$-cadherin and vimentin expression was increased with IL-6 treatment in HCCLM3 and SMMC-7721 cells as compared to the control group (absence of IL-6) in a dose-dependent manner (Fig. 1A). When the HCCLM3 and SMMC-7721 cells were treated with $100 \mathrm{ng} / \mathrm{ml}$ of IL-6 for 0, 24, and $48 \mathrm{~h}$, the data revealed that EMT had occurred in a time-dependent manner (Fig. 1B). In addition, we examined the expression of EMT markers in hepatocellular cells by immunofluorescence staining. After the HCCLM3 and SMMC-7721 cells were treated with IL-6 (100 ng/ml) for $48 \mathrm{~h}$, similar effects of E-cadherin, $\mathrm{N}$-cadherin and vimentin were observed (Fig. 1C). We next determined the mRNA expression levels of E-cadherin, $\mathrm{N}$-cadherin and vimentin in response to IL-6 $(100 \mathrm{ng} / \mathrm{ml})$ treatment for 0,24 and $48 \mathrm{~h}$ by quantitative RT-PCR. The mRNA levels of E-cadherin were significantly decreased in HCCLM3 and SMMC-7721 cells treated with IL-6 as compared to the control cells without IL-6 treatment. In contrast, the mRNA levels of $\mathrm{N}$-cadherin and vimentin were markedly increased in HCCLM3 and SMMC-7721 cells treated with IL-6 as compared to the control cells without IL-6 treatment (Fig. 1D). In summary, these results indicated that HCCLM3 and SMMC-7721 cells undergo EMT in response to IL-6 exposure, via downregulation of epithelial marker E-cadherin, and upregulation of mesenchymal markers $\mathrm{N}$-cadherin and vimentin. 
A

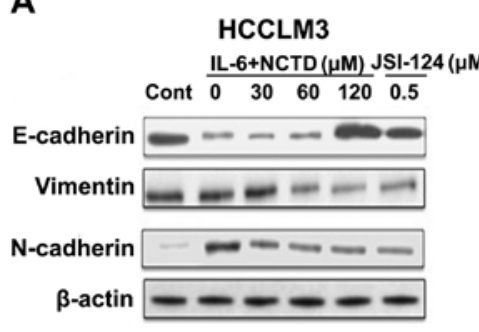

B

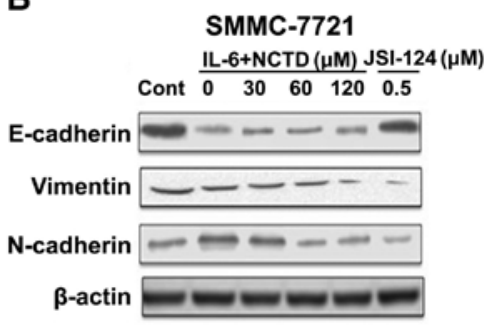

C
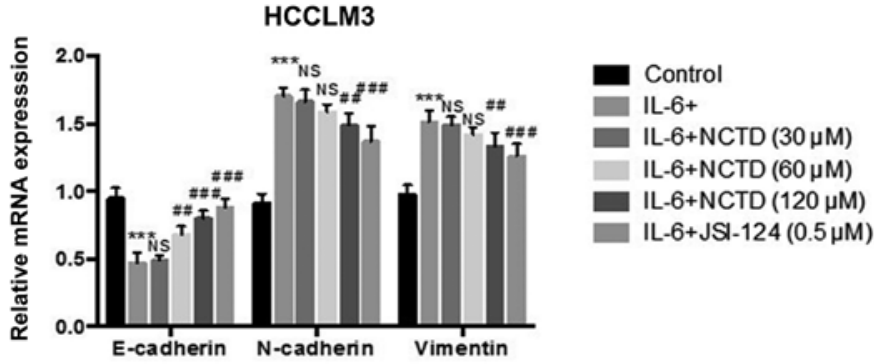

D

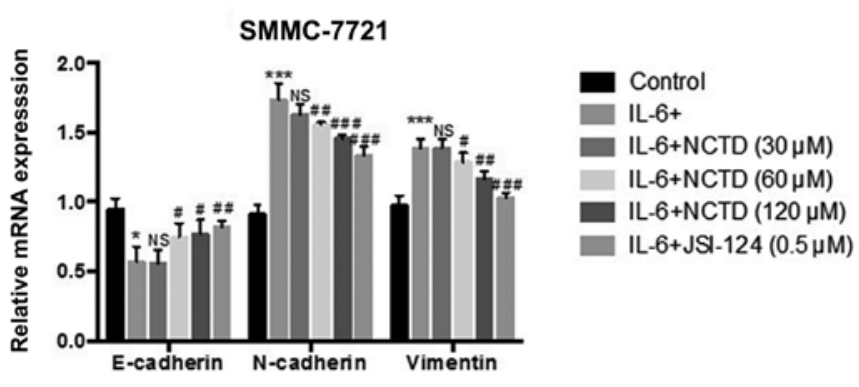

Figure 3. NCTD effectively blocks the IL-6 induced EMT in HCC cell lines. (A and B) After pretreatment with IL-6 (100 ng/ml) for 48 h, western blot analysis of E-cadherin, N-cadherin and vimentin protein expression was performed in HCCLM3 and SMMC-7721 cells after exposure to various concentrations of NCTD $(30,60$ and $120 \mu \mathrm{M})$ or JSI-124 $(0.5 \mu \mathrm{M})$ for $24 \mathrm{~h}$. (C and D) Cells were pretreated with IL-6 (100 ng/ml) for $48 \mathrm{~h}$ and the levels of E-cadherin, $\mathrm{N}$-cadherin and vimentin mRNA were determined by RT-PCR after exposure to various concentrations of NCTD (30, 60 and $120 \mu \mathrm{M})$ or JSI-124 ( $0.5 \mu \mathrm{M})$ for $24 \mathrm{~h}$ in HCCLM3 and SMMC-7721 cell lines. E-cadherin was upregulated, whereas N-cadherin and vimentin were downregulated following incubation with NCTD for $24 \mathrm{~h}$ as compared to the IL-6-treated group $\left(0 \mu \mathrm{M}\right.$ of NCTD). The mean values $\pm \mathrm{SD}, \mathrm{n}=3$ are provided. ${ }^{*} \mathrm{P}<0.05,{ }^{* *} \mathrm{P}<0.01,{ }^{* * *} \mathrm{P}<0.001 \mathrm{vs}$. the control group, NS P $>0.05,{ }^{\#} \mathrm{P}<0.05,{ }^{\# \# P} \mathrm{P}<0.01,{ }^{\# \#} \mathrm{P}<0.001$ vs. the IL-6 group). NCTD, norcantharidin; IL-6, interleukin 6; EMT, epithelial-mesenchymal transition; HCC, hepatocellular carcinoma.

IL-6-induced EMT is mediated by the activation of the JAK2/STAT3/TWIST pathway in HCC cells. JAK2/STAT3 signaling regulates different cellular functions, including the EMT process, in multiple cancer types. Herein, western blot results revealed that STAT3 was phosphorylated and activated to upregulate EMT transcription factor, TWIST, after IL-6 $(100 \mathrm{ng} / \mathrm{ml})$ treatment for the indicated durations $(0,24$ and $48 \mathrm{~h})$ in HCC cell lines HCCLM3 and SMMC-7721 (Fig. 2A-a and b). We hypothesized that JAK2/STAT3 signaling is required for IL-6-mediated EMT in HCC. To test this hypothesis, we used a JAK2/STAT3 specific inhibitor cucurbitacin I (JSI-124) to suppress the activation of STAT3. JSI-124 decreased STAT3 phosphorylation and TWIST expression induced by IL-6, respectively, in HCCLM3 and SMMC-7721 (Fig. 2A-c and d). We further examined the role of JSI-124 in IL-6-mediated EMT in HCCLM3 and SMMC-7721 cells. As observed, JSI-124 significantly reversed IL-6-mediated downregulation of E-cadherin, upregulation of $\mathrm{N}$-cadherin and vimentin, and decreased the mRNA levels of Twist (Fig. 2B).

We used Transwell assays to further investigate the invasive capacities of HCCLM3 and SMMC-7721 cells. IL-6 treatment with $100 \mathrm{ng} / \mathrm{ml}$ for $24 \mathrm{~h}$ enhanced cell invasiveness, whereas incubation with JSI-124 markedly suppressed the IL-6-induced increase of invaded cells. The results revealed that IL-6 enhanced the invasion abilities of HCCLM3 and SMMC-7721 cell lines, and that the JAK2/STAT3 inhibitor (JSI-124) suppressed IL-6-induced cell invasion (Fig. 2C and D). These findings demonstrated that upregulation of TWIST by STAT3 was required for IL-6-induced EMT and invasion in the HCCLM3 and SMMC-7721 cell lines.

Norcantharidin suppresses IL-6-induced EMT in HCC cells. To investigate the effect of NCTD treatment on IL-6-induced EMT in the HCCLM3 and SMMC-7721 cell lines, the cells were treated with $100 \mathrm{ng} / \mathrm{ml}$ of IL-6 for $48 \mathrm{~h}$. Western blot analysis revealed that the expression of epithelial marker, E-cadherin, was significantly increased, while mesenchymal markers, $\mathrm{N}$-cadherin and vimentin, were downregulated following incubation with various $\operatorname{NCTD}$ concentrations $(0,30$, 60 and $120 \mu \mathrm{M})$ for $24 \mathrm{~h}$ when compared to the IL-6-treated group ( $0 \mu \mathrm{M}$ of NCTD) (Fig. 3A and B). Furthermore, the relative mRNA levels of EMT markers from the NCTD groups after treatment with $100 \mathrm{ng} / \mathrm{ml}$ of IL- 6 for $48 \mathrm{~h}$, were analyzed 
A a

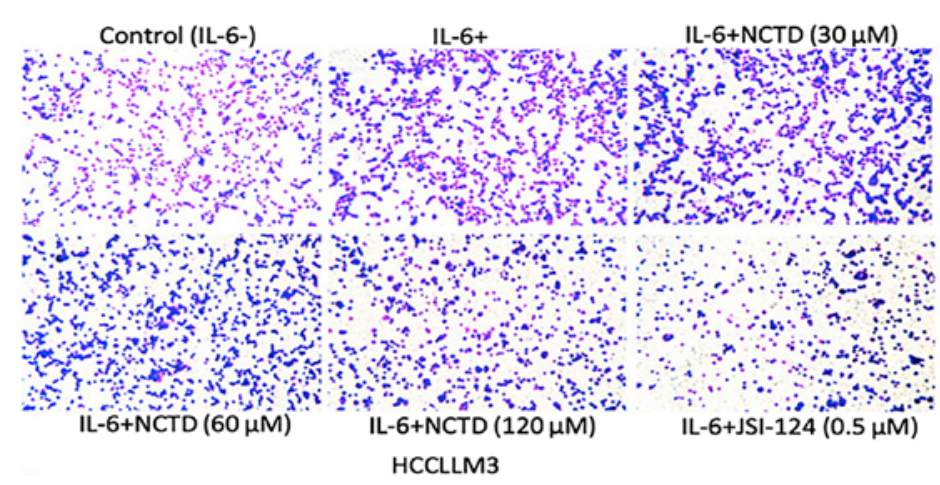

\section{B a}

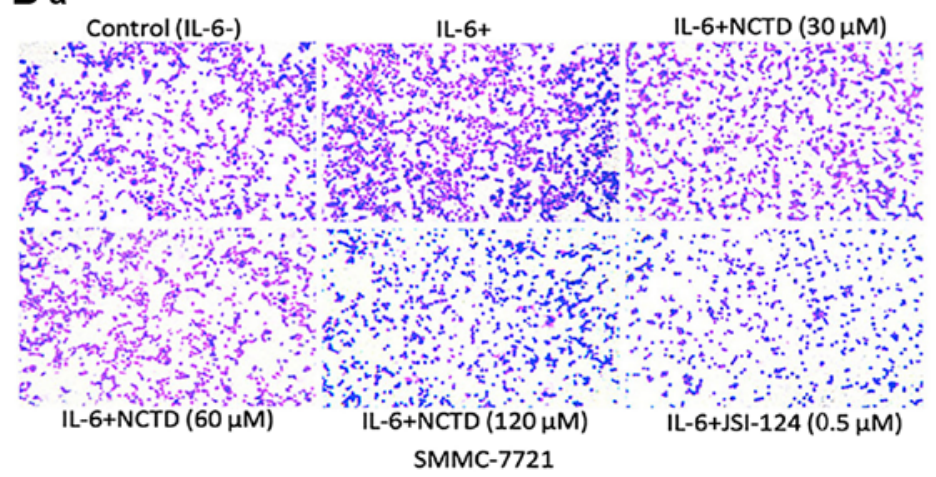

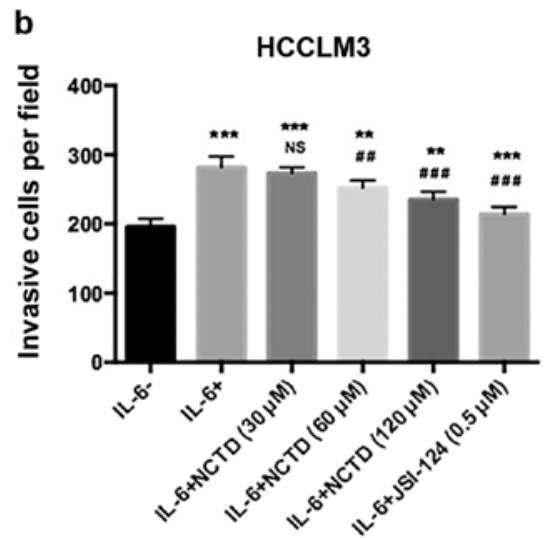

b

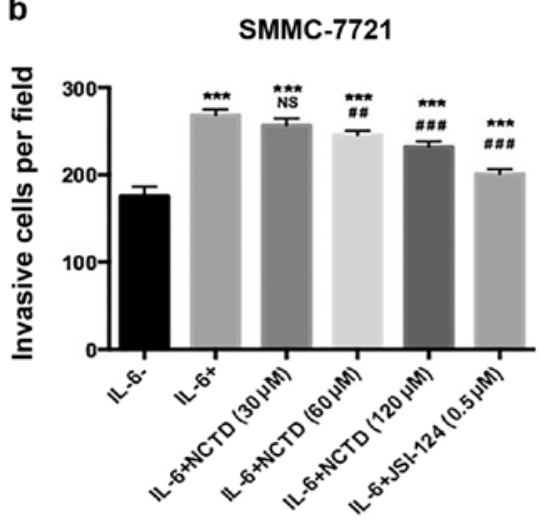

Figure 4. NCTD significantly suppresses the IL-6-induced invasiveness in HCC cell lines. (A-a and b and B-a and b) Cell invasive abilities were assessed by Transwell assay with different treatments in HCCLM3 and SMMC-7721 cells (magnification, x200). IL-6 significantly enhanced the invasive abilities after pretreatment (100 ng/ml of IL-6) for $48 \mathrm{~h}$. However, NCTD and JSI-124 decreased cell invasion after exposure to various concentrations of NCTD (30, 60 and $120 \mu \mathrm{M})$ and JSI-124 $(0.5 \mu \mathrm{M})$, for $24 \mathrm{~h}$. The mean values $\pm \mathrm{SD}, \mathrm{n}=3$ are provided. ${ }^{*} \mathrm{P}<0.05,{ }^{* *} \mathrm{P}<0.01,{ }^{* * * *} \mathrm{P}<0.001$ vs. the control group, NS $\mathrm{P}>0.05,{ }^{*} \mathrm{P}<0.05$, ${ }^{\# \#} \mathrm{P}<0.01,{ }^{\# \#} \mathrm{P}<0.001$ vs. the IL-6 group). NCTD, norcantharidin; IL-6, interleukin 6; HCC, hepatocellular carcinoma.

by quantitative RT-PCR. E-cadherin mRNA was suppressed after IL-6 treatment. However, this suppression was reversed by treatment with different NCTD concentrations (60 and $120 \mu \mathrm{M})$. In contrast, the relative levels of $\mathrm{N}$-cadherin and vimentin mRNA transcripts were increased after IL-6 treatment. However, this induction was prevented in the NCTD-treated (120 $\mu \mathrm{M})$ group (Fig. 3C and D). Collectively, the data demonstrated that NCTD treatment reversed the EMT process induced by IL-6 in the HCCLM3 and SMMC-7721 cell lines.

NCTD inhibits IL-6-induced cell invasion in HCC cells. The effects of NCTD on IL-6-induced cell invasion in the HCCLM3 and SMMC-7721 cell lines was further investigated by Transwell assays. The results revealed that IL-6 significantly enhanced the invasion ability of the two cell lines $(\mathrm{P}<0.001)$, whereas concomitant incubation with NCTD or JSI-124 suppressed the IL-6-induced invasion ability of the cells (Fig. 4). Thus, these findings demonstrated that NCTD suppressed IL-6-induced cellular invasiveness in a dose-dependent manner.

NCTD inhibits IL-6-induced EMT through the JAK/STAT3/TWIST signaling pathway in HCC cells. To explore the underlying mechanisms of the inhibitory activities of NCTD on IL-6-induced EMT, we determined its effect on the activation of the JAK/STAT3/TWIST pathway. The expression of JAK2, phosphorylated JAK2 (p-JAK2), STAT3, phosphorylated STAT3 (p-STAT3) and TWIST after IL-6 treatment in the different concentration NCTD-treated groups was analyzed by western blot analysis. Notably, the expression of p-JAK2 and p-STAT3 increased after exposure of HCCLM3 and SMMC-7721 cells to IL-6. Furthermore, the expression of p-JAK2 and p-STAT3 decreased after treatment with NCTD in a dose-dependent manner (Fig. 5A-a and b). Notably, the JAK2/STAT3 inhibitor JSI-124 significantly suppressed the p-JAK2 and p-STAT3 levels. Treatment with NCTD and JSI-124 also markedly decreased JAK2 and STAT3 phosphorylation induced by IL-6 (Fig. 5B).

Finally quantitative RT-PCR analysis revealed that the levels of JAK2 and STAT3 increased after IL-6 treatment, whereas after treatment with different concentrations of NCTD, the relative levels of JAK2, STAT3 and TWIST decreased in a dose-dependent manner in HCCLM3 and SMMC-7721 cells. Notably, the decrease in the expression of JAK2, STAT3 and TWIST with the NCTD $(30 \mu \mathrm{M})$ treatment was not in a statistically significant manner in the HCCLM3 cell lines (Fig. 5C). Moreover, NCTD $(120 \mu \mathrm{M})$ treatment resulted in a significant decrease of IL-6-mediated TWIST expression. Likewise the expression of TWIST in the two cell lines was compared to the suppression of JAK2/STAT3, suggesting that NCTD directly counteracted the IL-6-mediated induction of 
A a

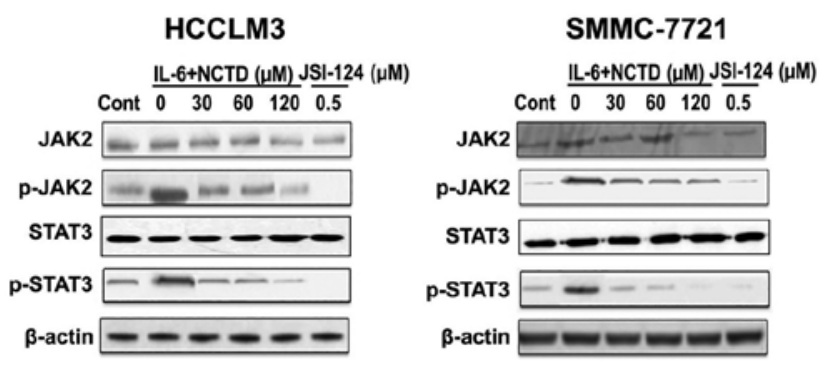

B a

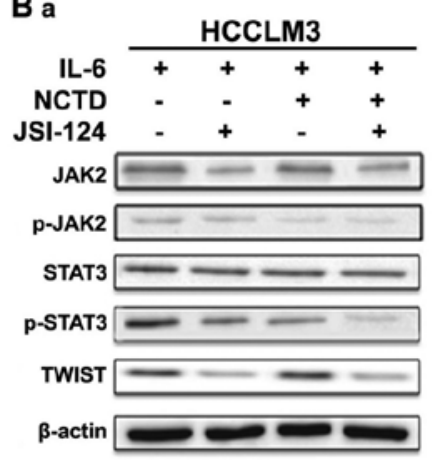

b

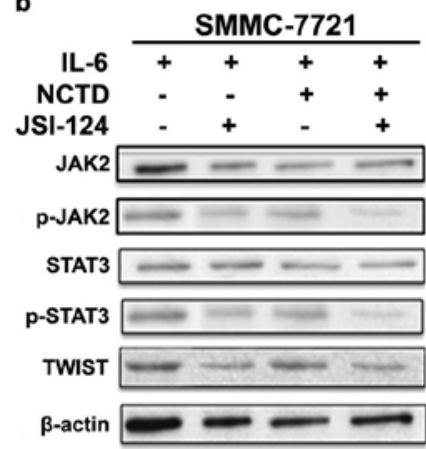

C a

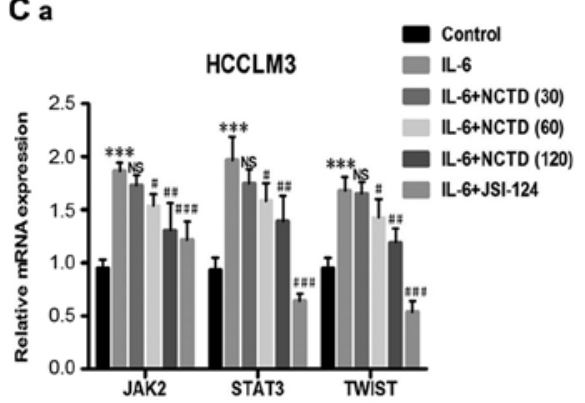

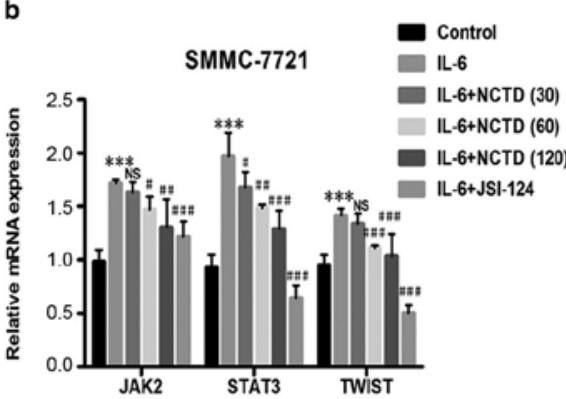

D a

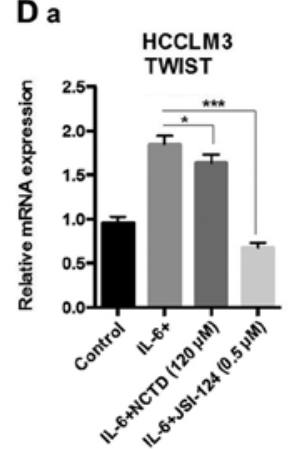

b

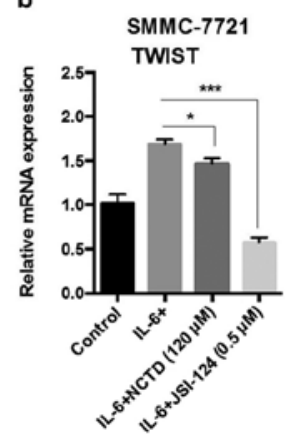

Figure 5. NCTD inhibits JAK2/STAT3/TWIST signaling in HCC cell lines. (A-a and b) After pretreatment with IL-6 (100 ng/ml) for $48 \mathrm{~h}$, western blot analysis of the protein expression of JAK2, phosphorylated JAK2 (p-JAK2), STAT3, and phosphorylated STAT3 (p-STAT3) was performed in HCCLM3 and SMMC-7721 cells after exposure to various concentrations of NCTD $(30,60$ and $120 \mu \mathrm{M})$ or JSI-124 $(0.5 \mu \mathrm{M})$ for $24 \mathrm{~h}$. (B-a and b) Cells were pretreated with IL-6 (100 ng/ml) for $48 \mathrm{~h}$, and the protein levels of JAK2, p-JAK2, STAT3, p-STAT3 and TWIST were determined by western blot analysis after exposure to NCTD $(120 \mu \mathrm{M})$ or JSI-124 $(0.5 \mu \mathrm{M})$ for $24 \mathrm{~h}$ in HCCLM3 and SMMC-7721 cell lines. (C-a and b) Cells were pretreated with IL-6 (100 ng/ml) for $48 \mathrm{~h}$, and the levels of JAK2, STAT3, TWIST mRNA were determined by RT-PCR after exposure to various concentrations of NCTD or JSI-124 (0.5 $\mu$ M) for $24 \mathrm{~h}$ in HCCLM3 and SMMC-7721 cell lines. (D-a and b) Cells were pretreated with IL-6 (100 ng/ml) for $48 \mathrm{~h}$, the mRNA levels of TWIST were determined by RT-PCR after exposure to NCTD $(120 \mu \mathrm{M})$ or JSI-124 $(0.5 \mu \mathrm{M})$ for $24 \mathrm{~h}$ in HCCLM3 and SMMC-7721 cell lines. (Mean values \pm SD, $\mathrm{n}=3$, are provided. ${ }^{*} \mathrm{P}<0.05,{ }^{* *} \mathrm{P}<0.01,{ }^{* * *} \mathrm{P}<0.001$ vs. the control group, NS $\mathrm{P}>0.05,{ }^{*} \mathrm{P}<0.05,{ }^{\# \#} \mathrm{P}<0.01,{ }^{\# \# \#} \mathrm{P}<0.001$ vs. the IL-6 group). NCTD, norcantharidin; JAK, Janus tyrosine kinase; STAT3, signal transducer and activator of transcription 3; IL-6, interleukin 6; HCC, hepatocellular carcinoma.

TWIST (Fig. 5D). In summary, these results demonstrated that NCTD inhibited IL-6-induced EMT via the downregulation of the TWIST transcription factor through JAK2/STAT3 signaling.

\section{Discussion}

EMT has been recognized as one of the universal mechanisms by which cancer cells acquire migratory and invasive capacity $(5,32-34)$. Invasion is a key step to progression toward a malignant phenotype, and occurs when tumor cells translocate from the relatively constrained initial neoplastic mass into neighboring host tissues. During the process of EMT, epithelial cells acquire a fibroblastoid appearance due to downregulation of epithelial markers and upregulation of mesenchymal markers, thus generating a migratory phenotype $(35,36)$. EMT which has been actively investigated in HCC (37-39), can be induced by numerous cytokines and growth factors, including IL-6 (40). Previous studies revealed that IL-6 induces a complex reciprocally regulated cytokine network in tumor cells which leads to the development of malignant and invasive tumors (40-42). IL-6 was shown to promote EMT changes in mesenchymal tumors from HCC patients as well as mesenchymal HCC cell lines (6). HCCLM3 and SMMC-7721 cell lines have highly invasive properties and were used in metastasis studies $(43,44)$. In this study, we established the IL-6-induced EMT model in HCC cell lines (HCCLM3 and SMMC-7721), and demonstrated that IL- 6 stimulated EMT in a time- and dose-dependent manner accompanied by downregulation of E-cadherin and upregulation of $\mathrm{N}$-cadherin and vimentin in HCCLM3 and SMMC-7722 cells. In addition, administration of IL-6 significantly enhanced the invasion potential of HCC cell lines as a result of EMT. Previous studies have suggested that IL- 6 can induce EMT changes by activating its downstream protein STAT3 within the tumor microenvironment, and plays an important role in the pathogenesis of human cervical carcinoma and breast cancer $(41,45)$. Whether STAT3 plays a role in EMT induction in human HCC still remains to be determined. JSI-124 is a selective inhibitor of JAK2/STAT3 and has been demonstrated to exert anti-proliferative and antitumor effects both in vitro and in vivo (46). In the present study, we found that the JAK2/STAT3 inhibitor (JSI-124) reversed the IL-6-induced EMT process and STAT3 phosphorylation in HCC cell lines, suggesting that JAK2/STAT3 may be involved in IL-6-induced EMT change and may be one of the mechanisms responsible for this change. Twist has been reported as a major transcriptional suppressor downregulating E-cadherin expression and thus resulting in initial EMT and ultimate tumor metastasis $(47,48)$. Emerging evidence suggests that STAT3 activation upregulates Twist expression $(17,41)$. 
Our data revealed that Twist, as an EMT activator, was significantly increased during EMT induction by IL-6. However, blocking STAT3 activation markedly suppressed the expression of Twist, confirming the regulation of Twist by STAT3.

In addition to EMT change, both HCCLM3 and SMMC-7721 cells after treatment with IL-6 gained invasive capacities. Using the cell line model, we investigated the effects of NCTD on IL-6induced EMT and cell invasion. The results revealed that NCTD hindered the upregulation of $\mathrm{N}$-cadherin and vimentin, and the downregulation of E-cadherin induced by IL-6 in HCCLM3 and SMMC-7721 cells, as well as the increased cell invasiveness induced by IL-6. IL-6-mediated activation of JAK2/ STAT3 implicated in EMT and metastasis, and inhibition of JAK2 or blockade of activated STAT3 significantly suppressed the EMT process, cell migration and invasion in colon and pancreatic cancer $(49,50)$. During the investigation process into the effects of NCTD on the levels of p-JAK2 and p-STAT3 in liver cancer cells after the treatment of IL-6, we found that NCTD significantly inhibited the activation of JAK2/ STAT3 induced by IL-6, although there was a slight variation in the levels of JAK2 in the presence of NCTD or JSI-124. The variation could be caused by different cell activity states. Moreover, we observed that the EMT-related transcription factor TWIST was downregulated after NCTD treatment, both at the mRNA and protein levels. All of these results clearly demonstrated that NCTD could block IL-6-induced EMT via the JAK2/STAT3/ TWIST signaling pathway, which is an important mechanism underlying HCC development and metastasis.

To conclude, IL- 6 promotes the EMT process during HCC development. NCTD can effectively target cell EMT by suppressing JAK2/STAT3/TWIST signaling under IL-6 stimulation. These findings warrant further assessment of NCTD, as an anticancer drug, in clinically relevant cancer models to explore its potential role in the treatment of HCC patients.

\section{Acknowledgements}

The present study was supported by the National Natural Science Foundation of China (Beijing, China; grant no. 81403346), the Natural Science Foundation of Shandong Province (Shandong, China; grant no. ZR2014HL095), and the China Postdoctoral Science Foundation (Beijing, China; grant no. 2014M550132).

\section{References}

1. Jemal A, Bray F, Center MM, Ferlay J, Ward E and Forman D: Global cancer statistics. CA Cancer J Clin 61: 69-90, 2011.

2. Wang $\mathrm{H}$ and Chen L: Tumor microenviroment and hepatocellular carcinoma metastasis. J Gastroenterol Hepatol 28 (Suppl 1): 43-48, 2013.

3. Thiery JP, Acloque H, Huang RY and Nieto MA: Epithelial-mesenchymal transitions in development and disease. Cell 139: 871-890, 2009.

4. Iwatsuki M, Mimori K, Yokobori T, Ishi H, Beppu T, Nakamori S, Baba $\mathrm{H}$ and Mori M: Epithelial-mesenchymal transition in cancer development and its clinical significance. Cancer Sci 101: 293-299, 2010

5. Kalluri R and Weinberg RA: The basics of epithelial-mesenchymal transition. J Clin Invest 119: 1420-1428, 2009.

6. Yamada S, Okumura N, Wei L, Fuchs BC, Fujii T, Sugimoto H, Nomoto S, Takeda S, Tanabe KK and Kodera Y: Epithelial to mesenchymal transition is associated with shorter disease-free survival in hepatocellular carcinoma. Ann Surg Oncol 21: 3882-3890, 2014.
7. Hussain SP and Harris CC: Inflammation and cancer: An ancient link with novel potentials. Int J Cancer 121: 2373-2380, 2007.

8. López-Novoa JM and Nieto MA: Inflammation and EMT: An alliance towards organ fibrosis and cancer progression. EMBO Mol Med 1: 303-314, 2009.

9. Rose-John S, Waetzig GH, Scheller J, Grötzinger J and Seegert D: The IL-6/sIL-6R complex as a novel target for therapeutic approaches. Expert Opin Ther Targets 11: 613-624, 2007.

10. Grivennikov SI and Karin M: Inflammatory cytokines in cancer: Tumour necrosis factor and interleukin 6 take the stage. Ann Rheum Dis 70 (Suppl 1): i104-i108, 2011.

11. Rokavec M, Wu W and Luo JL: IL6-mediated suppression of miR-200c directs constitutive activation of inflammatory signaling circuit driving transformation and tumorigenesis. Mol Cell 45: 777-789, 2012.

12. Porta C, De Amici M, Quaglini S, Paglino C, Tagliani F, Boncimino A, Moratti R and Corazza GR: Circulating interleukin- 6 as a tumor marker for hepatocellular carcinoma. Ann Oncol 19: 353-358, 2008.

13. Shackel NA, McCaughan GW and Warner FJ: Hepatocellular carcinoma development requires hepatic stem cells with altered transforming growth factor and interleukin-6 signaling. Hepatology 47: 2134-2136, 2008.

14. Wong VW, Yu J, Cheng AS, Wong GL, Chan HY, Chu ES, Ng EK, Chan FK, Sung JJ and Chan HL: High serum interleukin-6 level predicts future hepatocellular carcinoma development in patients with chronic hepatitis B. Int J Cancer 124: 2766-2770, 2009.

15. Yu H, Pardoll D and Jove R: STATs in cancer inflammation and immunity: A leading role for STAT3. Nat Rev Cancer 9: 798-809, 2009.

16. Colomiere M, Ward AC,Riley C, Trenerry MK,Cameron-Smith D, Findlay J, Ackland L and Ahmed N: Cross talk of signals between EGFR and IL-6R through JAK2/STAT3 mediate epithelial-mesenchymal transition in ovarian carcinomas. Br J Cancer 100: 134-144, 2009.

17. Chen W, Gao Q, Han S, Pan F and Fan W: The CCL2/CCR2 axis enhances IL-6-induced epithelial-mesenchymal transition by cooperatively activating STAT3-Twist signaling. Tumour Biol 36: 973-981, 2015.

18. Cheng GZ, Zhang WZ, Sun M, Wang Q, Coppola D, Mansour M, $\mathrm{Xu}$ LM, Costanzo C, Cheng JQ and Wang LH: Twist is transcriptionally induced by activation of STAT3 and mediates STAT3 oncogenic function. J Biol Chem 283: 14665-14673, 2008.

19. Ho YP, To KK, Au-Yeung SC, Wang X, Lin G and Han X: Potential new antitumor agents from an innovative combination of demethylcantharidin, a modified traditional Chinese medicine, with a platinum moiety. J Med Chem 44: 2065-2068, 2001.

20. Wang GS: Medical uses of mylabris in ancient China and recent studies. J Ethnopharmacol 26: 147-162, 1989.

21. Chen YN, Chen JC, Yin SC, Wang GS, Tsauer W, Hsu SF and Hsu SL: Effector mechanisms of norcantharidin-induced mitotic arrest and apoptosis in human hepatoma cells. Int J Cancer 100: 158-165, 2002.

22. Fan YZ, Zhao ZM, Fu JY, Chen CQ and Sun W: Norcantharidin inhibits growth of human gallbladder carcinoma xenografted tumors in nude mice by inducing apoptosis and blocking the cell cycle in vivo. Hepatobiliary Pancreat Dis Int 9: 414-422, 2010.

23. Fan YZ, Fu JY, Zhao ZM and Chen CQ: Inhibitory effect of norcantharidin on the growth of human gallbladder carcinoma GBC-SD cells in vitro. Hepatobiliary Pancreat Dis Int 6: 72-80, 2007.

24. Kok SH, Cheng SJ, Hong CY, Lee JJ, Lin SK, Kuo YS, Chiang CP and Kuo MY: Norcantharidin-induced apoptosis in oral cancer cells is associated with an increase of proapoptotic to antiapoptotic protein ratio. Cancer Lett 217: 43-52, 2005.

25. Yu CC, Ko FY, Yu CS, Lin CC, Huang YP, Yang JS, Lin JP and Chung JG: Norcantharidin triggers cell death and DNA damage through S-phase arrest and ROS-modulated apoptotic pathways in TSGH 8301 human urinary bladder carcinoma cells. Int J Oncol 41: 1050-1060, 2012.

26. Yang PY, Chen MF, Kao YH, Hu DN, Chang FR and Wu YC: Norcantharidin induces apoptosis of breast cancer cells: Involvement of activities of mitogen activated protein kinases and signal transducers and activators of transcription. Toxicol In Vitro 25: 699-707, 2011.

27. Huang Y, Liu Q, Liu K, Yagasaki K and Zhang G: Suppression of growth of highly-metastatic human breast cancer cells by norcantharidin and its mechanisms of action. Cytotechnology 59: 209, 2009. 
28. Chen YJ, Kuo CD, Tsai YM, Yu CC, Wang GS and Liao HF: Norcantharidin induces anoikis through Jun-N-terminal kinase activation in CT26 colorectal cancer cells. Anticancer Drugs 19: 55-64, 2008

29. Luan J, Duan H, Liu Q, Yagasaki K and Zhang G: Inhibitory effects of norcantharidin against human lung cancer cell growth and migration. Cytotechnology 62: 349-355, 2010.

30. Liu Q, Duan H, Luan J, Yagasaki K and Zhang G: Effects of theanine on growth of human lung cancer and leukemia cells as well as migration and invasion of human lung cancer cells Cytotechnology 59: 211-217, 2009.

31. Blaskovich MA, Sun J, Cantor A, Turkson J, Jove R and Sebti SM: Discovery of JSI-124 (cucurbitacin I), a selective Janus kinase/signal transducer and activator of transcription 3 signaling pathway inhibitor with potent antitumor activity against human and murine cancer cells in mice. Cancer Res 63: 1270-1279, 2003.

32. Thiery JP: Epithelial-mesenchymal transitions in tumour progression. Nat Rev Cancer 2: 442-454, 2002

33. Nieto MA: The ins and outs of the epithelial to mesenchymal transition in health and disease. Annu Rev Cell Dev Biol 27 347-376, 2011.

34. De Craene B and Berx G: Regulatory networks defining EMT during cancer initiation and progression. Nat Rev Cancer 13: 97-110, 2013

35. Yang $J$ and Weinberg RA: Epithelial-mesenchymal transition: At the crossroads of development and tumor metastasis. Dev Cell 14: 818-829, 2008

36. Nieto MA and Cano A: The epithelial-mesenchymal transition under control: Global programs to regulate epithelial plasticity. Semin Cancer Biol 22: 361-368, 2012.

37. Lee TK, Poon RT, Yuen AP, Ling MT, Kwok WK, Wang XH, Wong YC, Guan XY, Man K, Chau KL, et al: Twist overexpression correlates with hepatocellular carcinoma metastasis through induction of epithelial-mesenchymal transition. Clin Cancer Res 12: 5369-5376, 2006.

38. Lee TK, Man K, Poon RT, Lo CM, Yuen AP, Ng IO, Ng KT, Leonard W and Fan ST: Signal transducers and activators of transcription $5 \mathrm{~b}$ activation enhances hepatocellular carcinoma aggressiveness through induction of epithelial-mesenchymal transition. Cancer Res 66: 9948-9956, 2006.

39. Giannelli G, Bergamini C, Fransvea E, Sgarra C and Antonaci S: Laminin-5 with transforming growth factor-betal induces epithelial to mesenchymal transition in hepatocellular carcinoma. Gastroenterology 129: 1375-1383, 2005.
40. Yadav A, Kumar B, Datta J, Teknos TN and Kumar P: IL-6 promotes head and neck tumor metastasis by inducing epithelial-mesenchymal transition via the JAK-STAT3-SNAIL signaling pathway. Mol Cancer Res 9: 1658-1667, 2011.

41. Sullivan NJ, Sasser AK, Axel AE, Vesuna F, Raman V, Ramirez N, Oberyszyn TM and Hall BM: Interleukin-6 induces an epithelial-mesenchymal transition phenotype in human breast cancer cells. Oncogene 28: 2940-2947, 2009.

42. Lederle W, Depner S, Schnur S, Obermueller E, Catone N, Just A, Fusenig NE and Mueller MM: IL-6 promotes malignant growth of skin SCCs by regulating a network of autocrine and paracrine cytokines. Int J Cancer 128: 2803-2814, 2011.

43. Li Y, Tian B, Yang J, Zhao L, Wu X, Ye SL, Liu YK and Tang ZY: Stepwise metastatic human hepatocellular carcinoma cell model system with multiple metastatic potentials established through consecutive in vivo selection and studies on metastatic characteristics. J Cancer Res Clin Oncol 130: 460-468, 2004.

44. Yao J, Liang L, Huang S, Ding J, Tan N, Zhao Y, Yan M, Ge C, Zhang Z, Chen T, et al: MicroRNA-30d promotes tumor invasion and metastasis by targeting Galphai2 in hepatocellular carcinoma. Hepatology 51: 846-856, 2010.

45. Miao JW, Liu LJ and Huang J: Interleukin-6-induced epithelial-mesenchymal transition through signal transducer and activator of transcription 3 in human cervical carcinoma. Int $\mathrm{J}$ Oncol 45: 165-176, 2014.

46. Su Y, Li G, Zhang X, Gu J, Zhang C, Tian Z and Zhang J: JSI-124 inhibits glioblastoma multiforme cell proliferation through $\mathrm{G}(2) / \mathrm{M}$ cell cycle arrest and apoptosis augment. Cancer Biol Ther 7: 1243-1249, 2008.

47. Liu AN, Zhu ZH, Chang SJ and Hang XS: Twist expression associated with the epithelial-mesenchymal transition in gastric cancer. Mol Cell Biochem 367: 195-203, 2012.

48. Sasaki K, Natsugoe S, Ishigami S, Matsumoto M, Okumura H, Setoyama T, Uchikado Y, Kita Y, Tamotsu K, Sakamoto A, et al: Significance of Twist expression and its association with E-cadherin in esophageal squamous cell carcinoma. J Exp Clin Cancer Res 28: 158, 2009.

49. Bromberg J and Wang TC: Inflammation and cancer: IL-6 and STAT3 complete the link. Cancer Cell 15: 79-80, 2009.

50. Huang C, Yang G, Jiang T, Zhu G, Li H and Qiu Z: The effects and mechanisms of blockage of STAT3 signaling pathway on IL-6 inducing EMT in human pancreatic cancer cells in vitro. Neoplasma 58: 396-405, 2011. 